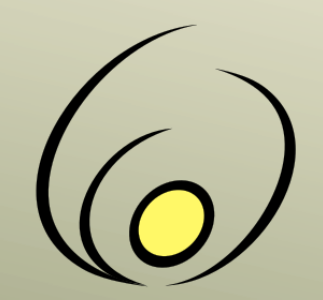

Fórum de Pró-Reitores de Extensão das Instituições Públicas de Educação Superior Brasileiras

\section{Revista Brasileira de Extensão Universitária}

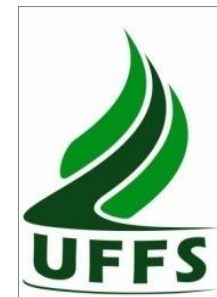

v. 12 , n. 1, p. 13-22, jan.-abr. 2021

e-ISSN 2358-0399

\title{
Cuidados farmacêuticos em instituições de ação social: A extensão como estratégia de ensino e promoção da saúde
}

\author{
Jacqueline Aparecida Eidam Horst ${ }^{1}$, Paulo Roberto Parecy Junior ${ }^{1}$, Luciana Erzinger Alves de \\ Camargo $^{1,2}$, Tatiana Herrerias ${ }^{1,2}$, Daniel de Paula ${ }^{3}$
}

\begin{abstract}
Resumo: O cuidado farmacêutico é uma prática profissional, centrada no usuário, que visa à promoção e recuperação da saúde por meio da abordagem farmacoterapêutica. O objetivo desse estudo foi relatar ações extensionistas realizadas em instituições de ação social, na forma de cuidados farmacêuticos prestados por estudantes e professores do curso de Farmácia da Universidade Estadual do Centro-Oeste do Paraná. Um total de 36 estudantes e cinco professores realizaram, entre abril e junho de 2019, consultas farmacêuticas com 57 pessoas assistidas por duas instituições sociais situadas na região urbana do município de Guarapuava/PR. Foi realizado rastreamento de saúde com testes de glicemia capilar e aferição da pressão arterial. A associação entre comorbidades, faixa etária e o consumo de medicamentos foi analisada pelo teste de Pearson. Os estudantes concederam depoimentos através de entrevista aberta, os quais foram interpretados por análise de discurso temática. Do total de 57 consultas farmacêuticas, 47 (82,5\%) pessoas receberam aconselhamento, 45 (78,9\%) passaram por intervenções, incluindo mudanças no horário de administração dos medicamentos e orientações sobre possíveis interações com alimentos, e $23(40,3 \%)$ receberam encaminhamento médico. Os depoimentos dos estudantes foram categorizados semanticamente em relação à teoria versus prática, formação profissional e vínculo com o paciente. Assim, foi possível reconhecer a saúde como condição resultante do contexto social, suscitando o elo entre promoção da saúde e as condições de vida. O processo de aprendizagem requer do estudante observações próprias da realidade, com atitudes reflexivas que decorrem da inserção de atividades de extensão e pesquisa como metodologia de ensino.
\end{abstract}

Palavras-chave: Cuidados de Saúde; Assistência Farmacêutica; Uso Racional de Medicamentos; Educação em Saúde

\section{Pharmaceutical care in social institutions: extension as a strategy for learning and health promotion}

Abstract: Pharmaceutical care is a professional, user-centered practice that aims to promote and recover health using the pharmacotherapeutic approach. This study aimed to report extensionist actions in social care institutions, as pharmaceutical care provided by students and professors of the Pharmacy course at the Universidade Estadual do Centro-Oeste do Paraná (Paraná State, Brazil). A total of 36 students and five professors conducted pharmaceutical consultations from April to June 2019 with 57 people assisted by two social care institutions located in the urban region of the city of Guarapuava/PR. Health screening was performed with capillary blood glucose tests and blood pressure measurements. The association between comorbidities, age groups, and drug consumption was analyzed by Pearson's test. The students gave testimonies through the open interviews, and the speeches were interpreted by thematic speech analysis. Out of a total of 57 consultations, $47(82.5 \%)$ people received pharmaceutical counseling, 45 (78.9\%) went through pharmaceutical interventions, including changes in medication administration schedule and guidance on possible food interactions, and $23(40.3 \%)$ received a medical referral. The students' testimonies were categorized semantically concerning theory versus practice, professional training, and bond with the patient. Thus, it was possible to recognize health as a condition resulting from the social context, raising the link between health promotion and living conditions. The learning process requires students' observations of reality, with reflective attitudes that result from the insertion of extension and research activities as teaching methodologies.

Keywords: Health Care; Pharmaceutical Services; Rational Drug Utilization; Health Education
Originais recebidos em 04 de agosto de 2020

Aceito para publicação em

12 de dezembro de 2020

1 Departamento de Farmácia, Setor de Ciências da Saúde, Universidade Estadual do Centro-Oeste do Paraná UNICENTRO, Guarapuava/PR

\section{2}

Curso de Farmácia, Centro Universitário UniGuairacá, Guarapuava/PR

3

Departamento de Farmácia, Setor de Ciências da Saúde, Universidade Estadual do Centro-Oeste do Paraná UNICENTRO, Guarapuava/PR (autor para correspondência) ddepaula@unicentro.br 


\section{Introdução}

Cuidado em saúde é o conjunto de ações e serviços ofertados à comunidade que considera a autonomia do ser humano, sua singularidade, e o contexto real em que vive, sendo realizado por meio de atividades de promoção, proteção e recuperação da saúde (Portaria de Consolidação $n^{\circ} 2$ do Ministério da Saúde, de 28 de setembro de 2017; Roosli et al., 2020). Centrada no usuário, o cuidado farmacêutico é uma prática profissional que visa à adesão ao tratamento farmacológico, a resolução de problemas da farmacoterapia, e o uso racional dos medicamentos, por meio da provisão de uma variedade de serviços farmacêuticos. Esses serviços objetivam a educação e o rastreamento em saúde, a dispensação de medicamentos, e o manejo de problemas de saúde autolimitados (Ministério da Saúde, 2014).

As mudanças na formação acadêmica dos profissionais de saúde, ampliando os cenários de aprendizado e adequando os currículos às necessidades sociais são essenciais no contexto atual de saúde do Brasil, e incrementam as competências dos estudantes (Panúncio-Pinto et al., 2015; Hermann et al., 2018). Entretanto, o ensino tradicional de Farmácia concentrou-se, por muito tempo, no modelo Biomédico/Hospitalocêntrico/ Flexneriano, pautado na retenção de informações e repetição de conteúdos básicos, sem contemplar a resolução de problemas, uma competência essencial para a prática dos cuidados farmacêuticos (Pagliosa \& Ros, 2008; Almeida Filho, 2010).

De acordo com as diretrizes curriculares de 2017 (Ministério da Educação, 2017), o profissional farmacêutico da atualidade deve ter formação humanista, crítica, reflexiva e generalista, capacitando-o para o trabalho nos diferentes níveis de complexidade do sistema de saúde e, sobretudo, assumindo o cuidado em saúde como prática profissional.

Com o pressuposto de pensar o aprendizado como processo de construção coletiva, a extensão universitária possibilita transformações da realidade e, especificamente na área da saúde, propicia que as ações de cuidado resultem em reflexões críticas, que emergem dos questionamentos entre prática e teoria (Silva et al., 2013). Neste sentido, a formação pautada em ações extensionistas deve ser conduzida pela interação dialógica, interdisciplinaridade e interprofissionalidade, de forma a manter o elo entre ensino-pesquisa-extensão e promover impacto na formação do estudante e na transformação social (Ministério da Educação, 2018).

As ações extensionistas em saúde são, geralmente, desenvolvidas em comunidades em vulnerabilidade social, que possuem determinantes sociais da saúde (DSS) que influenciam a ocorrência de problemas de saúde, precipitando fatores de risco à população (Buss \& Filho, 2007; Carrapato et al., 2017). Esses fatores de riscos, associados diretamente aos fatores de vulnerabilidade, como renda familiar, condições precárias de domicílio, nível de escolaridade, além do próprio lugar que vivem, revelam uma situação de desigualdade em contextos de negação dos direitos sociais (Lima, 2016; Sousa et al., 2017).

Neste cenário, a promoção da saúde pode ser entendida no que se refere às ações sobre os DSS, conduzidas ao processo de capacitação da comunidade para atuar na melhoria da sua qualidade de vida (QV) e saúde (Malta et al., 2018). Mais adiante, as ações de promoção da saúde podem impulsionar movimentos de participação social no processo de formulação e gestão das políticas públicas de saúde, direcionando-as para o cumprimento efetivo das diretrizes e dos princípios do Sistema Único de Saúde (SUS): universalidade, integralidade, equidade, descentralização, participação e controle social (Portaria no 2.761 do Ministério da Saúde, de 19 de novembro de 2013).

Com o desafio de implementar as transformações na concepção da profissão farmacêutica, a criação de ambientes e práticas pedagógicas que proporcionem situações próximas da realidade atual, tanto social como do sistema de saúde, são uma necessidade desafiadora à academia. Dessa forma, o objetivo desse estudo foi 
relatar ações extensionistas realizadas em instituições de ação social, na forma de cuidados farmacêuticos prestados por estudantes e professores do curso de Farmácia da Universidade Estadual do Centro-Oeste do Paraná (UNICENTRO/PR).

\section{Metodologia}

Trata-se de um relato de experiência, com coleta de dados por meio de consulta farmacêutica em duas instituições de ação social no município de Guarapuava/PR, acrescido da abordagem da experiência de estudantes de Farmácia da UNICENTRO/PR. As ações descritas ocorreram no período de abril a junho de 2019 nas instalações das instituições sociais, contando com a participação de 36 estudantes do $4^{\circ}$ e $5^{\circ}$ ano, supervisionados por cinco professores. Em 2019 as instituições atenderam 155 pessoas e o projeto contou com 57 participantes.

Este estudo apresenta o resultado das ações extensionistas de um projeto integrado ensino-pesquisa-extensão na área de Assistência Farmacêutica, aprovado em 2019 e com duração de três anos, que visa auxiliar na implantação das novas diretrizes curriculares (Ministério da Educação, 2017) e curricularização da extensão no curso de Farmácia da UNICENTRO/PR.

Com população estimada em 182 mil habitantes, Guarapuava está localizada na região centro-sul paranaense, que engloba 29 municípios com cerca de 544 mil habitantes. Embora seja a maior mesorregião em termos geográficos, é a que possui os menores índices de desenvolvimento humano do estado (Instituto Paranaense de Desenvolvimento Social [IPARDES], 2020). As instituições de ação social, local do estudo, estão localizadas na região urbana, sendo integradas ao Sistema Único de Assistência Social (SUAS). Juntas, desenvolvem projetos e programas que visam o atendimento a famílias em situação de vulnerabilidade e risco pessoal e social.

O critério de inclusão dos estudantes foi: estar regularmente matriculado nas disciplinas relacionadas aos cuidados farmacêuticos. Os depoimentos dos estudantes foram coletados com base nos critérios: ter participado do treinamento inicial, de todas as atividades extensionistas, e ter sido aprovado nas disciplinas envolvidas. Os critérios de inclusão da população atendida foram: pessoas com idade $\geq 18$ anos e enquadrados em situação de vulnerabilidade social pelo cadastro anual das instituições.

As consultas farmacêuticas foram realizadas pelos estudantes, sob a supervisão de um professor, usando a Ficha Farmacoterapêutica (Ministério da Saúde, 2014). As consultas compreenderam escuta ativa, identificação de necessidades, análise da situação, tomada de decisões, definição de condutas, documentação e avaliação. Para tal, os estudantes receberam orientação prévia sobre habilidades de comunicação e conhecimentos de farmacoterapia por meio de simulações com casos clínicos.

Durante as consultas foram realizados teste de glicemia capilar (Sociedade Brasileira de Diabetes [SBD], 2017) e aferição da pressão arterial (Malachias et al., 2016) para fins de rastreamento em saúde. Após 4 semanas da primeira consulta foi realizada a devolutiva aos participantes. Esta ação envolveu aconselhamento e orientação sobre medicamentos e problemas de saúde, intervenções farmacêuticas e encaminhamentos aos serviços de saúde. O perfil epidemiológico de medicamentos foi elaborado com base na classificação farmacológica da Anatomic Therapeutic Chemical (ATC) (Collaborating Centre for Drug Statistics Methodology [CCDSM], 2016). A associação entre o consumo de medicamentos, a idade e os indicadores de saúde, pressão arterial e glicemia foi feita pelo coeficiente de correlação de Pearson.

Ao final de cada atividade, estudantes e professores se reuniam para avaliar o andamento das consultas. Nesse momento coletou-se os depoimentos dos estudantes por meio da técnica de entrevista aberta (Guerra, 2014), os quais foram convidados a falar livremente sobre o significado da experiência extensionista em sua formação. 
Os depoimentos foram identificados com o pseudônimo "estudante $\mathrm{n}^{\circ}$., Ano". Para a interpretação dos resultados usou-se a análise de conteúdo temática (Bardin, 2009), organizada em 3 polos: i) pré-análise com recurso de leitura flutuante e identificação de pontos de análise, categorização e inferência qualitativa, ii) exploração do material, por meio de categorização semântica dos elementos presentes nos depoimentos, com realização dos recortes e correções necessários e, iii) inferência e interpretações qualitativas com base nas categorias estabelecidas. Este estudo foi aprovado pelo Comitê de Ética em Pesquisa da Unicentro/PR, sob CAAE 07005118.2.0000.0106, e protocolo de aprovação n 3.407.022, de 29 de março de 2019.

\section{Resultados e Discussão}

\section{Cuidados Farmacêuticos como Estratégia de Promoção da Saúde}

Foram realizadas consultas farmacêuticas com 57 pessoas assistidas, com idade entre 24 e 86 anos, as quais foram abordadas durante os grupos de trabalho semanais que ocorrem nas instituições sociais. Durante as consultas realizadas pelos estudantes, com supervisão dos professores, foram obtidas diversas informações relacionadas à saúde e ao uso de medicamentos dos participantes. A partir dessas informações foi possível traçar o perfil da população em relação ao consumo de medicamentos e a promoção da saúde. A maior parte das pessoas é do sexo feminino, com 54 participantes (94,7\%). A respeito das doenças, 21 (36,8\%) relataram ter hipertensão arterial e 7 (12,3\%) diabetes mellitus. Com relação aos hábitos de saúde, 10 (17,2\%) participantes eram tabagistas, 9 (15,5\%) ingerem bebidas alcoólicas e 27 (47,4\%) praticavam atividade física regularmente (Tabela 1 ).

Quando se analisa uso de medicamentos, 11 (19,3\%) pessoas não faziam uso, 10 (17,5\%) usavam 1 medicamento, 24 (42,1\%) 2 a 4 medicamentos e $12(21,1 \%)$ usavam 5 ou mais medicamentos, caracterizando polifarmácia. Os fármacos mais usados atuam sobre o sistema nervoso (16,6\%), sistema cardiovascular (15,8\%), trato alimentar e metabolismo (7,9\%) e sistema musculoesquelético (3,3\%). Os demais fármacos citados (56,3\%) pertenciam à classe dos que afetam o sistema respiratório, antianêmicos, agentes hormonais, vitaminas, entre outros (Tabela 2).

Os cuidados farmacêuticos realizados neste estudo incluíram rastreamento em saúde com medição da glicemia capilar e pressão arterial; manejo de problemas de saúde autolimitados; revisão da farmacoterapia, com intervenções e encaminhamentos quando necessário, além de aconselhamentos farmacêuticos relativo a questões de saúde. O aconselhamento farmacêutico é uma ação de educação em saúde individualizada, na qual esse profissional orienta o paciente a obter o melhor aproveitamento da farmacoterapia visando não somente a cura, mas também a melhora na QV (Ministério da Saúde, 2014). Dos pacientes atendidos, 47 (82,5\%) receberam aconselhamento farmacêutico relativo a hábitos de alimentação saudável, prática de exercícios físicos e riscos da automedicação.

Um total de 45 (78,9\%) pessoas receberam intervenções farmacêuticas visando otimizar a eficácia da farmacoterapia, aumentar a adesão ao tratamento e/ou reduzir a ocorrência de efeitos indesejados. Tais intervenções incluíram mudanças no horário de administração dos medicamentos e orientações sobre possíveis interações com alimentos. Em 23 (40,3\%) casos, quando foram detectados problemas como erros de posologia, reações adversas graves, falta de eficácia e/ou interações medicamentosas com significado clínico, foram realizados encaminhamentos médicos. Estes foram feitos pelos estudantes, por meio de formulário próprio, com carimbo e assinatura do professor responsável com registro no Conselho Regional de Farmácia. Nesses casos, os pacientes foram orientados a agendar consulta de retorno com o médico sobre a necessidade de alterações na farmacoterapia. 
Tabela 1. Perfil do consumo de medicamentos da população assistida pelas instituições sociais. GuarapuavaPR, 2019.

\begin{tabular}{|c|c|c|c|c|c|c|c|c|c|c|}
\hline \multirow{3}{*}{$\begin{array}{l}\text { Variável } \\
\text { Sexo }\end{array}$} & \multicolumn{8}{|c|}{ Número de medicamentos } & \multirow{3}{*}{$\mathrm{n}$} & \multirow{3}{*}{$\begin{array}{c}\text { Freq. } \\
(\%)\end{array}$} \\
\hline & \multicolumn{2}{|c|}{0} & \multicolumn{2}{|c|}{1} & \multicolumn{2}{|c|}{2 a 4} & \multicolumn{2}{|c|}{5 ou mais } & & \\
\hline & $\mathrm{n}$ & (\%) & $\mathrm{n}$ & (\%) & $\mathrm{n}$ & (\%) & $\mathrm{n}$ & (\%) & & \\
\hline Masculino & 2 & 66,7 & 0 & 0 & 1 & 33,3 & 0 & 0 & 3 & 5,3 \\
\hline Feminino & 9 & 16,7 & 10 & 18,5 & 23 & 42,6 & 12 & 22,2 & 54 & 94,7 \\
\hline \multicolumn{11}{|l|}{ Faixa etária } \\
\hline 18 a 40 anos & 5 & 27,8 & 3 & 16,7 & 9 & 50,0 & 1 & 5,5 & 18 & 31,6 \\
\hline 41 a 59 anos & 4 & 19,1 & 5 & 23,8 & 8 & 38,1 & 4 & 19,0 & 21 & 36,8 \\
\hline 60 anos ou mais & 2 & 11,1 & 2 & 11,1 & 7 & 38,9 & 7 & 38,9 & 18 & 31,6 \\
\hline Tabagista & 0 & 0 & 2 & 20,0 & 5 & 50,0 & 3 & 30,0 & 10 & 17,5 \\
\hline Não-tabagista & 11 & 23,4 & 8 & 17,0 & 19 & 40,4 & 9 & 19,2 & 47 & 82,5 \\
\hline \multicolumn{11}{|c|}{ Ingere bebidas alcoólicas } \\
\hline Sim & 3 & 33,3 & 1 & 11,1 & 4 & 44,4 & 1 & 11,2 & 9 & 15,8 \\
\hline Não & 8 & 16,7 & 9 & 18,8 & 20 & 41,6 & 11 & 22,9 & 48 & 84,2 \\
\hline \multicolumn{11}{|c|}{ Atividade física regular } \\
\hline $\operatorname{Sim}$ & 4 & 14,8 & 4 & 14,8 & 14 & 51,9 & 5 & 18,5 & 27 & 47,4 \\
\hline Não & 7 & 23,3 & 6 & 20 & 10 & 33,3 & 7 & 23,3 & 30 & 52,6 \\
\hline \multicolumn{11}{|l|}{ Diabetes mellitus } \\
\hline $\operatorname{Sim}$ & 2 & 28,6 & 0 & 0 & 1 & 14,3 & 4 & 57,1 & 7 & 12,3 \\
\hline Não & 9 & 18,0 & 10 & 20,0 & 23 & 46,0 & 8 & 16,0 & 50 & 87,7 \\
\hline \multicolumn{11}{|l|}{ Hipertensão arterial } \\
\hline Sim & 1 & 4,8 & 3 & 14,3 & 10 & 47,6 & 7 & 33,3 & 21 & 36,8 \\
\hline Não & 10 & 27,8 & 7 & 19,4 & 14 & 38,9 & 5 & 13,9 & 36 & 63,2 \\
\hline TOTAL & 11 & 19,3 & 10 & 17,5 & 24 & 42,1 & 12 & 21,1 & 57 & 100 \\
\hline
\end{tabular}

O aumento do consumo de medicamentos pode estar associado a vários fatores, como a presença de múltiplas comorbidades, maior expectativa de vida e maior quantidade de fármacos disponíveis no mercado (Nascimento et al., 2017; Santos et al., 2019). A correlação entre as variáveis sexo, faixa etária e condições de saúde dos participantes em função da quantidade de medicamentos em uso pode ser observada na Tabela 1.

O teste de Pearson revelou uma correlação positiva baixa $(0,311)$ entre polifarmácia e a idade, e correlação negativa entre a polifarmácia e a glicemia capilar $(-0,104)$ e a pressão arterial $(-0,049)$.

A prevalência de polifarmácia (21,1\%) nessa população foi semelhante à encontrada na Escócia, de 20,8\% (Guthrie et al., 2015). Porém, foi superior aos valores observados nas cinco regiões do Brasil (9,4\%) (Nascimento et al., 2017) e na Alemanha (10,0\%) (Grimmsmann \& Himmel, 2009). 
Tabela 2. Principais fármacos usados pela população assistida pelas instituições sociais de acordo com a Anatomic Therapeutic Chemical. Guarapuava-PR, 2019.

\begin{tabular}{|c|c|c|c|c|}
\hline Fármaco & Grupo principal & Subgrupo terapêutico & $\mathrm{n}$ & $\begin{array}{l}\text { Frequência } \\
\text { (\%) }\end{array}$ \\
\hline Paracetamol & \multirow{3}{*}{ Sistema nervoso } & \multirow{3}{*}{ Analgésicos } & 10 & \multirow{3}{*}{16,6} \\
\hline Dipirona & & & 8 & \\
\hline $\begin{array}{c}\text { Dipirona } \\
\text { associação* }\end{array}$ & & & 7 & \\
\hline Losartana & \multirow{4}{*}{$\begin{array}{c}\text { Sistema } \\
\text { cardiovascular }\end{array}$} & $\begin{array}{l}\text { Fármacos que atuam } \\
\text { sobre o sistema Renina } \\
\text { Angiotensina }\end{array}$ & 11 & \multirow{4}{*}{15,9} \\
\hline Hidroclorotiazida & & Diurético & 5 & \\
\hline Atenolol & & Betabloqueador & 4 & \\
\hline Sinvastatina & & Modificador de lipídeos & 4 & \\
\hline Omeprazol & \multirow{2}{*}{$\begin{array}{l}\text { Trato alimentar e } \\
\text { metabolismo }\end{array}$} & $\begin{array}{l}\text { Bloqueador de bomba de } \\
\text { próton }\end{array}$ & 8 & \multirow{2}{*}{7,9} \\
\hline Metformina & & Antidiabético & 4 & \\
\hline Ibuprofeno & $\begin{array}{c}\text { Sistema } \\
\text { musculoesquelético }\end{array}$ & $\begin{array}{l}\text { Anti-inflamatório e } \\
\text { antirreumático }\end{array}$ & 5 & 3,3 \\
\hline Subtotal & & & 66 & 43,7 \\
\hline TOTAL & & & 151 & 100 \\
\hline
\end{tabular}

*Associação de dipirona com orfenadrina e cafeína.

Do total de 18 idosos, 7 (38,9\%) são polimedicados, prevalência superior aos 28\% encontrados em idosos em Goiânia (Silveira et al., 2014), aos 32,7\% em aposentados no Rio de Janeiro (Rozenfeld et al., 2008), e aos 32\% em idosos em Florianópolis (Pereira et al., 2017). Os impactos clínicos da polifarmácia em idosos são relacionados a fatores tais como risco de efeitos adversos, interações medicamentosas, uso de medicamentos potencialmente inapropriados (MPI), problemas de adesão ao tratamento, admissão hospitalar, e quedas (Cadogan et al., 2016; Marques et al., 2018).

Quando o assunto é promoção da saúde, surge o desafio de compreendê-la a partir da subjetividade de seu significado. A partir de então, reconhece-se a saúde como resultante de fatores interligados com o contexto social, político e econômico, suscitando o elo entre promoção de saúde e as condições de vida (Silva et al., 2013; Sousa et al., 2017). A educação em saúde é considerada uma das principais ações de promoção da saúde e se revela de suma importância na prevenção e reabilitação de doenças, além de despertar a cidadania e a responsabilidade social relacionada à saúde (Stotz, 1993; Costa et al., 2015).

\section{Impacto da extensão na formação do farmacêutico}

A extensão universitária caracteriza-se como processo educativo, cultural e científico, fundamentado na articulação do ensino e da pesquisa e na viabilização da relação transformadora entre universidade e 
comunidade. Este processo viabiliza a troca de saberes acadêmico e popular, tendo como consequência a produção do conhecimento em confronto com a realidade, a democratização do conhecimento acadêmico e a participação efetiva da comunidade na atuação da universidade (Costa et al., 2015).

O processo formativo em saúde, desenvolvido com base em ações extensionistas, induz a produção de novas práticas de cuidado integral e a formação integral, aquela focada não apenas na aprendizagem técnica, mas na ética, responsabilidade cidadã e compromisso social (Silva et al., 2013).

Nesta atividade extensionista participaram 36 estudantes de Farmácia, sendo 20 do $4^{\circ}$ ano, e 16 do $5^{\circ}$ ano, com idade entre 20 e 26 anos, sendo 30 (83\%) do sexo feminino. Após os atendimentos, reuniam-se professores e estudantes para discussões, quando era solicitado aos estudantes que comentassem sobre suas impressões a respeito das atividades realizadas. Os estudantes relatavam quais foram suas principais dificuldades e as correlações observadas entre os aspectos teóricos aprendidos em sala de aula e a realidade vivenciada durante os atendimentos. Analisando os depoimentos dos estudantes sobre o impacto das atividades extensionistas na formação profissional, destacam-se os seguintes fragmentos:

"Além de conseguir interagir com os pacientes, consegui também usar dos meus conhecimentos adquiridos em sala de aula para ajudá-los. O método utilizado de colocar os alunos em contato com a população foi essencial para que pudéssemos compreender melhor nossa função como futuros farmacêuticos, não apenas na teoria. Com a prática, pude enxergar o quanto o trabalho do farmacêutico é essencial. (Estudante 1 do $4^{\circ}$ ano)."

"Na sala de aula se tem a teoria como base, que também é importante, mas fica difícil a visualização de como funciona, do que nos espera, pois cada paciente é de um jeito e cada um tem suas queixas e problemas tendo, portanto, a necessidade de praticar a teoria que nos foi exposta, a prática me fez ter mais confiança, o contato com o paciente me fez ter mais segurança e experiência em todos os aspectos (Estudante 2 do $4^{\circ}$ ano)."

"Aprendemos muito com essa atividade pois exigiu que nos concentrássemos em casos diferentes, - fazendo com que estudássemos a doença, o tratamento, os medicamentos utilizados pelo paciente e as interações entre todos eles, bem como, o uso dos mesmos, sendo necessários ou não. A teoria nos dá a base sobre como os medicamentos agem e para que eles servem, mas a prática possibilita ver como é feito o uso de tais medicamentos, como o uso incorreto influencia no tratamento, como a polifarmácia muitas vezes pode não ajudar o paciente. É uma experiência válida e que agrega muito ao aluno (Estudante 3 do $4^{\circ}$ ano)."

"Foi uma experiência que me fez amadurecer muito como profissional. O contato com o paciente te estimula cada vez estudar mais, para passar a informação de forma correta quando você for questionado, porque você nunca sabe o que o paciente vai querer saber. A principal diferença da teoria com a prática foi como se portar perante o paciente como explicar de uma maneira que ele entenda o que você está falando. E na teoria isso era muito mais simples, mas na prática foi um choque. Eu consegui associar muitos conhecimentos da teoria com a prática e aprendi muito mais sobre interações medicamentosas, como tratar o paciente e sobre as classes de medicamentos (Estudante 5 do $4^{\circ}$ ano)."

"Foi um desafio adequar uma metodologia e até mesmo o vocabulário, para atingir a todos, com diferentes idades, mas que com certeza agregou muito para a nossa profissão. Hoje, trabalhando em farmácia eu entendo ainda mais a importância de criar um vínculo com os pacientes e clientes com quem trabalho (estudante 1 do $5^{\circ}$ ano)."

Os elementos presentes nos depoimentos dos estudantes foram categorizados semanticamente em: i) relação teoria versus prática, ii) formação profissional e iii) vínculo com o paciente. Percebe-se que o processo formativo através das ações extensionistas desenvolve-se a partir das relações dialógicas entre pessoas de diferentes saberes e nos confrontos entre teoria e prática, que se estabelecem no aprender e fazer nas vivências extensionistas, pois as relações subjetivas afetam a produção do cuidado integral (Silva et al., 2013).

Considera-se relevante pensar a educação, a partir de Paulo Freire, sustentada pelo diálogo como compromisso humanista e conscientizador. Nessa perspectiva, compreende-se o processo de educação como 
complexo e multidimensional, com capacidade de contribuição para que o "educando torne-se sujeito de seu próprio desenvolvimento, diante da presença orientadora que deve ter o educador" (Freire, 2011; Ribeiro et al., 2018).

A pedagogia da problematização é um exemplo de como é possível estabelecer um processo de ensinoaprendizagem cooperativo, coletivo, no qual professor e estudante descobrem juntos alternativas e conhecimentos novos, em que não há uma superioridade daquele que educa frente àquele que está sendo educado. Também colabora no estabelecimento de vínculos entre profissionais de saúde e comunidade, já que as pessoas ficam mais próximas umas das outras e trabalham de modo solidário (Martins \& Stauffer, 2007).

A extensão precisa ser vista como um processo de formação que permite ao estudante o contato com a realidade externa aos muros da universidade. É preciso reconhecer que na formação acadêmica ocorrem mudanças, e que ela precisa ser entendida como crítica e plural, não podendo se restringir à simples transmissão do conhecimento restrito à sala de aula (Saldan \& Santos, 2019).

A prática extensionista se coloca como campo de aprendizagem não só dos estudantes, mas dos professores e demais envolvidos, pois, para se aprender, é preciso vivenciar o mundo, e não só ter teorias (Silva et al., 2013).

A partir das percepções dos estudantes nas atividades desenvolvidas nas instituições sociais foi possível observar que a extensão é uma estratégia fundamental para inserção do estudante na realidade profissional, e para auxiliar a correlação entre os conhecimentos teóricos de sala de aula e a prática profissional. A prática assistencial agrega à formação profissional, possibilitando abordagens trans e interdisciplinares na formação acadêmica, e promove mudanças de consciência social. Entretanto, a utilização da extensão como prática pedagógica é um desafio, pois os cursos de Farmácia, tradicionalmente, sempre foram conteudistas e tecnicistas. Desta forma, a melhora da formação farmacêutica, além de mudanças curriculares, requer nova postura dos professores e estudantes, mais voltada para o desenvolvimento de habilidades de identificação e análise das necessidades de saúde da população.

\section{Conclusões}

Esse estudo evidenciou a extensão como estratégia de ensino e promoção da saúde em ações voltadas a comunidades vulneráveis, apontando a extensão como processo educativo, viabilizador de uma relação transformadora entre a universidade e a comunidade. Os cuidados farmacêuticos prestados durante a ação extensionista possibilitaram reconhecer a saúde como condição resultante do contexto social, suscitando o elo entre promoção da saúde e as condições de vida. Neste sentido, o processo de aprendizagem requer do estudante observações próprias da realidade, com atitudes reflexivas que decorrem da inserção de atividades de extensão e pesquisa como metodologia de ensino. Através deste estudo, provoca-se uma discussão em relação à formação do profissional farmacêutico. Transformações na formação acadêmica requerem, além de mudanças curriculares, uma nova postura do estudante e professor. Consequentemente, faz-se necessária a criação de ações integradoras de ensino-pesquisa-extensão, que sejam capazes de impactar a formação do estudante e promover a transformação social.

\section{Contribuição de cada autor}

J.A.E.H. e P.R.P.J. realizaram a análise e interpretação dos dados e a redação do artigo. L.E.A.C. participou da redação do artigo e revisão crítica. T.H. contribuiu com a interpretação dos dados, redação e revisão crítica. D.D.P. foi o coordenador e responsável pela concepção e planejamento do projeto, revisão crítica e aprovação final. Todos os autores participaram das atividades de consulta farmacêutica e coleta de dados. 


\section{Referências}

Almeida Filho, N. (2010). Reconhecer Flexner: Inquérito sobre produção de mitos na educação médica no Brasil contemporâneo. Cadernos de Saúde Pública, 26(12), 2234-2249.

Bardin, L. (2009). Análise de conteúdo. Lisboa: Edições 70.

Buss, P. M., \& Filho, A. P. (2007). A Saúde e seus Determinantes Sociais. PHYSIS: Revista de Saúde Coletiva, 17(1), 77-93.

Cadogan, C.A., Ryan, C., \& Hughes, C.M. (2016). Appropriate polypharmacy and medicine safety: When many is not too many. Drug Safety, 39(2), 109-116.

Carrapato, P., Correia, P., \& Garcia, B. (2017). Determinante da saúde no Brasil: A procura da equidade na saúde. Saúde e Sociedade, 26(3), 676-689.

Collaborating Centre for Drug Statistics Methodology (2016). Anatomical Therapeutic Chemical (ATC) Classification Index, 2016 Oslo: WHO - CCDSM. Recuperado de https://www.whocc.no/atc ddd index/

Costa, J. M. B. S., Silva, V. L., Samico, I. C., \& Cesse, E. A. P. (2015). Desempenho de intervenções de saúde em países da América Latina: Uma revisão sistemática. Saúde Debate, 39(Especial), 307-319.

Freire, P. (2011). Pedagogia da autonomia: Saberes necessários à prática educativa. São Paulo: Paz e Terra.

Grimmsmann, T., \& Himmel, W. (2009). Polypharmacy in primary care practices: An analysis using a large health insurance database. Pharmacoepidemiology Drug Safety, 18(12), 1206-1213.

Guerra, E. L. A. (2014). Manual de Pesquisa Qualitativa. Belo Horizonte: Grupo Ănima Educação.

Guthrie, B., Makubate, B., Hernandez-Santiago, V., \& Dreischulte, T. (2015). The rising tide of polypharmacy and drugdrug interactions: Population database analysis 1995-2010. BMC Medicine, 13(74), 1-10.

Hermann A. P., Lacerda, M. R., Nascimento, J. D., Gomes, I. M., \& Zatoni, D. C. P. (2018). Enhancing the process of teaching and learning homecare. Revista Brasileira de Enfermagem, 71(1), 156-162.

Instituto Paranaense de Desenvolvimento Social (IPARDES). (2020). Caderno Estatístico Município de Guarapuava. Curitiba: IPARDES 50p. Recuperado de http://www.ipardes.pr.gov.br/Pagina/Cadernos-municipais

Lima, F. A. (2016). Territórios de vulnerabilidade social: Construção metodológica e aplicação em Uberlândia-MG. (Dissertação de Mestrado). Universidade Federal de Uberlândia-UFU, Uberlândia, Brasil. Recuperado de https://repositorio.ufu.br/bitstream/123456789/19449/1/TerritoriosVulnerabilidadeSocial.pdf

Malachias, M. V. B., Souza, W. K. S. B., Plavnik, F. L., Rodrigues, C. I. S., Brandão, A. A., Neves, M. F. T., ... \& Moreno Júnior, H. (2016). VII Diretriz Brasileira de Hipertensão Arterial. Arquivos Brasileiros de Cardiologia, 107(3 Supl. 3), 1103.

Malta, D. C., Reis A. A. C., Jaime, P. C., Morais Neto, O. L., Silva, M. M. A., \& Akerman, M. (2018). O SUS e a Política Nacional de Promoção da Saúde: Perspectiva resultados, avanços e desafios em tempos de crise. Ciência \& Saúde Coletiva, 23(6),1799-1809.

Marques, G. F. M., Rezende, D. M. R. P., Silva, I. P., Souza, P. C., Barbosa, S. R. M., Penha, R. M., \& Polisel, C. G. (2018). Polifarmácia e medicamentos potencialmente inapropriados para idosos na enfermagem gerontológica. Revista Brasileira de Enfermagem, 71(5), 2440-2446.

Martins, C. M., \& Stauffer, A. B. (2007). Educação e saúde. Rio de Janeiro: EPSJV/FIOCRUZ.

Ministério da Educação (2017). Resolução № 6, de 19 de outubro de 2017 (Institui as Diretrizes Curriculares Nacionais do Curso de Graduação em Farmácia e dá outras providências). Diário Oficial da União, s.1, p.30.

Ministério da Educação (2018). Resolução № 7, de 18 de dezembro de 2018 (Diretrizes para a Extensão na Educação Superior Brasileira). Diário Oficial da União, s.1, p.49. 
Ministério da Saúde (2014). Serviços farmacêuticos na atenção básica à saúde: Cuidado farmacêutico na atenção básica. Caderno 1. Brasília: Ministério da Saúde.

Ministério da Saúde (2017). Portaria de Consolidação No. 2, de 28 de setembro de 2017. Diário Oficial da União, s.1, p.61.2017.

Nascimento, R. C. R. M., Álvares, J., Guerra Junior, A. A., Gomes, I. C., Silveira, M. R., Costa, E. A., ... \& Acurcio, F. A. (2017). Polifarmácia: Uma realidade na atenção primária do Sistema Único de Saúde. Revista de Saúde Pública, 51(2), 1s-12s

Pagliosa, F. L., \& Ros, M. A. (2008). O Relatório Flexner: Para o bem e para o mal. Revista Brasileira de Educação Médica, 32(4), 492-499.

Panúncio-Pinto, M. P., Rodrigues, M. L. V., \& Fiorati, R. C. (2015). Novos cenários de ensino: A comunidade e o território como espaços privilegiados de formação de profissionais da saúde. Medicina (Ribeirão Preto), 48(3), 257-64.

Pereira, K. G., Peres, M. A., lop, D., Boing, A. C., Boing, A. F., Aziz, M., \& D’orsi, E. (2017). Polifarmácia em idosos: Um estudo de base populacional. Revista Brasileira de Epidemiologia, 20(2), 335-344.

Ribeiro, M. R. F., Mendes, F. F. F., \& Silva, E. A. (2018). Curricularização da extensão em prol de uma universidade socialmente referenciada. Revista Conexão UEPG, 4(3), 334-342.

Roosli, A. C. B. S., Palma, C. M. S., \& Ortolan, M. L. M. (2020). Sobre o cuidado na saúde: Da assistência ao cidadão à autonomia de um sujeito. Psicologia USP, 31, e180145.

Rozenfeld, S., Fonseca, M. J. M., \& Acurcio, F. A. (2008). Drug utilization and polypharmacy among the elderly: a survey in Rio de Janeiro City, Brazil. Revista Panamericana de Salud Publica, 23(1), 34-43.

Saldan, P. C., \& Santos, E. M. (2019). A curricularização da extensão na UNICENTRO: Trajetória, possibilidades e desafios. In: A. Kolody, \& D. J. A. N. Belém (Orgs.), Extensão em perspectivas. (pp. 185-202). Guarapuava: UNICENTRO.

Santos, L. F., Morais, A. E., Furtado, A. B., Pinto, B. N. S. L., Martins, K. R. S., Alves, E. B. \& Aguiar, T. L. (2019). Pharmacovigilance of polypharmacy and adverse drug reactions in hospitalized elderly in a university hospital in Manaus, Amazonas. Vigilância Sanitária em Debate, 7(4), 41-4.

Silva, A. F. L., Ribeiro, C. D. M., \& Silva Júnior, A. G. (2013). Pensando extensão universitária como campo de formação em saúde: Uma experiência na Universidade Federal Fluminense, Brasil. Interface - Comunicação, Saúde, Educação, $17(45), 371-384$.

Silveira, E. A, Dalastra, L., \& Pagotto, V. (2014). Polifarmácia, doenças crônicas e marcadores nutricionais em idosos. Revista Brasileira de Epidemiologia, 17(4), 818-29.

Sociedade Brasileira de Diabetes (SBD). (2017). Diretrizes da Sociedade Brasileira de Diabetes 2017-2018. São Paulo: Clannad.

Sousa, I. V., Brasil C. C. P., Silva, R. M., Vasconcelos, D. P., Silva, K. A., Bezerra, I. N., \& Finan, T. J. (2017). Diagnóstico participativo para identificação de problemas de saúde em comunidade em situação de vulnerabilidade social. Ciência \& Saúde Coletiva, 22(12), 3945-3954.

Stotz, E. N. (1993). Enfoques sobre educação e saúde. In V. V. Valla, \& E. N. Stotz (Orgs.). Participação popular, Educação e Saúde: Teoria e prática. (pp. 11-22). Rio de Janeiro: Relume-Dumará.

Como citar este artigo:

Horst, J. A. E., Parecy Junior, P R., de Camargo, L. E. A., Herrerias, T., \& de Paula, D. (2021). Cuidados farmacêuticos em instituições de ação social: A extensão como estratégia de ensino e promoção da saúde. Revista Brasileira de Extensão Universitária, 12(1), 1322. https://periodicos.uffs.edu.br/index.php/RBEU/article/view/11655/pdf 